\title{
Elastic surface waves and the structure of liquid surfaces
}

\author{
C. F. Tejero and M. J. Rodriguez \\ Departamento de Termologia, Facultad de Fisica, Universidad Complutense, Madrid 3, Spain \\ M. Baus \\ Laboratoire Chimie-Physique II, Association Euratom-Etat Belge, Université Libre de Bruxelles, \\ B-1050 Brussels, Belgium \\ (Received 12 July 1983)
}

\begin{abstract}
The high-frequency elastic behavior of fluid surfaces is studied from first principles. The general dispersion equation for elastic surface waves thus obtained reveals the influence of the surface structure on Rayleigh's surface waves. New types of surface waves are also found. Their existence depends crucially on the presence of nonvanishing surface excess elastic moduli and hence these waves are inaccessible to the macroscopic theory of elasticity. The experimental observation of these elastic surface waves could provide new information about the structure of the liquid surface.
\end{abstract}

\section{INTRODUCTION}

The liquid surface and, more generally, fluid-fluid interfaces are typical examples of nonuniform fluids. While the macroscopic theory of surface effects in fluids is already more than one century old, ${ }^{1}$ its microscopic foundation via statistical-mechanical methods is a fairly recent development. Until recently, the statistical-mechanical approach to nonuniform fluids ${ }^{2}$ has been mainly concerned with the static equilibrium properties of the liquid-vapor ${ }^{3}$ and the liquid-solid ${ }^{4}$ interface. The first studies of the dynamical properties of fluid interfaces by statistical methods have appeared in the literature only recently. In a first group of studies ${ }^{5}$ Bedeaux, Oppenheim, and Ronis have used a linear response theory in order to develop a statistical-mechanical basis for their earlier phenomenological treatment ${ }^{6}$ of the hydrodynamical equations of systems with an interface. In these phenomenological studies ${ }^{6}$ the interface region was considered as a singular two-dimensional phase endowed with physical properties distinct from those of the bulk phases. The singular surface properties could subsequently be recovered from the microscopic theory ${ }^{5}$ via the introduction of a surface multipole expansion using surfacebounded generalized functions (distributions). In a second group of studies ${ }^{7}$ Jhon, Desai, and Dahler have stressed the relation between the dynamics of the liquid surface and the theory of broken symmetries. In these studies the surface properties were extracted from the Mori-Zwanzig equations for the broken symmetry variables via the introduction of a surface projection operator. In a third group of studies ${ }^{8}$ we have applied the Mori-Zwanzig method directly to the nonuniform fluid and extracted interfacial equations by integrating the generalized hydrodynamic equations of the nonuniform fluid through the interface region from one bulk phase to the other while considering all spatial gradients except the normal ones to be slowly varying within the interfacial region. A number of other related studies should also be mentioned. ${ }^{9}$ In either case $^{5-9}$ the complexity of the results obtained is fairly large and based on a series of uncontrolled approximations. At present we feel that very little more can be learned by continuing these purely theoretical developments since they present problems (e.g., the gradient expansion) which have not been solved yet even for the bulk-phase equations. What is needed, in our opinion, are a few simple consequences of these theories which in principle could be checked experimentally. In this spirit we have investigated the consequences of the surface structure (i.e., its intrinsic diffuse nature ${ }^{2}$ as opposed to the steplike discontinuity assumed in the phenomenological theories ${ }^{1}$ ) on the dispersion relation for the various surface waves by assuming $a$ priori that the corrections due to this surface structure are small. Here we will consider the highfrequency elastic surface waves while the study of the low-frequency hydrodynamic waves is deferred to a later study.

In Sec. II we briefly recall the exact evolution equations for the conserved densities obtained by applying the Mori-Zwanzig method to a nonuniform fluid as explained in detail elsewhere. ${ }^{8(c)}$ In Sec. III we compute the highfrequency limit of these equations and obtain the basic wave equations of the theory of elasticity of nonuniform fluids. We then specialize these equations in Sec. IV to the particular case of a liquid-vapor system with a planar equilibrium interface. In Sec. $V$ we study the plane-wave solutions of these equations and obtain the general dispersion relation for the elastic surface waves on a free liquid surface. This dispersion equation is then studied in a few limiting cases while our conclusions are summarized in Sec. VI.

\section{GENERAL EVOLUTION EQUATIONS FOR NONUNIFORM FLUIDS}

Elsewhere ${ }^{8(\mathrm{c})}$ we have derived the evolution equations for the nonequilibrium statistical averages (denoted by \langle\rangle$)$ of the five conserved densities of a simple fluid of particles of mass $m$. These formally exact equations have been obtained through a straightforward extension of the 
Mori-Zwanzig projection-operator method to the case of fluids whose equilibrium state is nonuniform and anisotropic such as is required for the subsequent description of fluid interfaces. To this end we took as basic dynamical variables the number density $\hat{n}(\vec{x}, t)$, the momentum density $\widehat{g}_{\alpha}(\overrightarrow{\mathrm{x}}, t)(\alpha=1,2,3)$, and that part $\hat{e}_{n}(\overrightarrow{\mathrm{x}}, t)$ of the energy density $\hat{e}(\vec{x}, t)$ which is thermodynamically independent of the number density [i.e., $\left\langle\hat{e}_{n}(\vec{x}, t) \hat{n}\left(\vec{x}^{\prime}, t\right)\right\rangle_{0}=0$, where \langle\rangle$_{0}$ denotes the equilibrium ensemble average]. The microscopic definition of these variables will not be repeated here but can be found elsewhere. ${ }^{8(c)}$ The study of the linearized nonequilibrium deviations from the nonuniform equilibrium state can then be most conveniently studied in terms of the density deviation $\delta n(\vec{x}, t)=\langle\hat{n}(\vec{x}, t)\rangle-\rho(\vec{x})$, of the velocity field $u_{\alpha}(\vec{x}, t)=\left\langle\hat{g}_{\alpha}(\vec{x}, t)\right\rangle / m \rho(\vec{x})$, and of the deviation of the internal energy

$$
\delta \epsilon(\overrightarrow{\mathrm{x}}, t)=\left[\left\langle\widehat{e}_{n}(\overrightarrow{\mathrm{x}}, t)\right\rangle-\left\langle\widehat{e}_{n}(\overrightarrow{\mathrm{x}}, t)\right\rangle_{0}\right] / \rho(\overrightarrow{\mathrm{x}}),
$$

where $\rho(\vec{x})=\langle\hat{n}(\vec{x}, t)\rangle_{0}$ is the nonuniform equilibrium density profile. With this choice of variables the exact evolution equations can be written in the form of generalized linearized hydrodynamic equations of the following Fourier-transformed form $\left[A(\omega)=\int_{0}^{\infty} d t A(t) \exp (i \omega t)\right.$; $A(t=0)=0]:$

$$
\begin{aligned}
& -i \omega \delta n(\overrightarrow{\mathrm{x}}, \omega)+\nabla_{\beta}\left[\rho(\overrightarrow{\mathrm{x}}) u_{\beta}(\overrightarrow{\mathrm{x}}, \omega)\right]=0, \\
& -i \omega m \rho(\overrightarrow{\mathrm{x}}) u_{\alpha}(\overrightarrow{\mathrm{x}}, \omega)+k_{B} T \rho(\overrightarrow{\mathrm{x}}) \nabla_{\alpha} \int d \overrightarrow{\mathrm{x}}^{\prime} K_{n n}\left(\overrightarrow{\mathrm{x}}, \overrightarrow{\mathrm{x}}^{\prime}\right) \delta n\left(\overrightarrow{\mathrm{x}}^{\prime}, \omega\right)+\frac{1}{k_{B} T} \int d \overrightarrow{\mathrm{x}}^{\prime}\left\langle\hat{\mathrm{g}}_{\alpha}(\overrightarrow{\mathrm{x}}) \psi(\omega) \hat{\mathrm{g}}_{\beta}\left(\overrightarrow{\mathrm{x}}^{\prime}\right)\right\rangle_{0} u_{\beta}\left(\overrightarrow{\mathrm{x}}^{\prime}, \omega\right) \\
& +\frac{1}{k_{B} T^{2}} \int d \overrightarrow{\mathrm{x}}^{\prime}\left\langle\hat{\mathrm{g}}_{\alpha}(\overrightarrow{\mathrm{x}})\left[-\delta \hat{e}_{n}\left(\overrightarrow{\mathrm{x}}^{\prime}\right)+\psi(\omega) \delta \hat{\hat{e}}_{n}\left(\overrightarrow{\mathrm{x}}^{\prime}\right)\right]\right\rangle_{0} \delta T\left(\overrightarrow{\mathrm{x}}^{\prime}, \omega\right)=\left\langle\psi(\omega) \hat{\mathrm{g}}_{\alpha}(\overrightarrow{\mathrm{x}})\right\rangle_{0}, \\
& -i \omega \rho(\overrightarrow{\mathrm{x}}) \delta \epsilon(\overrightarrow{\mathrm{x}}, \omega)+\frac{1}{k_{B} T^{2}} \int d \overrightarrow{\mathrm{x}}^{\prime}\left\langle\delta \hat{e}_{n}(\overrightarrow{\mathrm{x}}) \psi(\omega) \delta \hat{\hat{e}}_{n}\left(\overrightarrow{\mathrm{x}}^{\prime}\right)\right\rangle_{0} \delta T\left(\overrightarrow{\mathrm{x}}^{\prime}, \omega\right) \\
& +\frac{1}{k_{B} T} \int d \overrightarrow{\mathrm{x}}^{\prime}\left\langle\delta \hat{\hat{e}}_{n}(\overrightarrow{\mathrm{x}})\left[-\hat{\mathrm{g}}_{\alpha}\left(\overrightarrow{\mathrm{x}}^{\prime}\right)+\psi(\omega) \hat{\mathrm{g}}_{\alpha}\left(\overrightarrow{\mathrm{x}}^{\prime}\right)\right]\right\rangle_{0} u_{\alpha}\left(\overrightarrow{\mathrm{x}}^{\prime}, \omega\right)=\left\langle\psi(\omega) \delta \hat{\hat{e}}_{n}(\overrightarrow{\mathrm{x}})\right\rangle_{0},
\end{aligned}
$$

where $\delta T(\overrightarrow{\mathrm{x}}, t)$ denotes the deviation of the local nonequilibrium temperature from its uniform equilibrium value $T$. This additional variable is obtained from the internal energy as

$$
\begin{aligned}
& \delta T(\overrightarrow{\mathrm{x}}, t)=k_{B} T^{2} \int d \overrightarrow{\mathrm{x}}^{\prime} K_{e_{n} e_{n}}\left(\overrightarrow{\mathrm{x}}, \overrightarrow{\mathrm{x}}^{\prime}\right) \rho\left(\overrightarrow{\mathrm{x}}^{\prime}\right) \delta \epsilon\left(\overrightarrow{\mathrm{x}}^{\prime}, t\right), \\
& \rho(\overrightarrow{\mathrm{x}}) \delta \epsilon(\overrightarrow{\mathrm{x}}, t)=\frac{1}{k_{B} T^{2}} \int d \overrightarrow{\mathrm{x}}^{\prime} G_{e_{n} e_{n}}\left(\overrightarrow{\mathrm{x}}, \overrightarrow{\mathrm{x}}^{\prime}\right) \delta T\left(\overrightarrow{\mathrm{x}}^{\prime}, t\right),
\end{aligned}
$$

a definition which differs slightly from the one used previously. ${ }^{8(c)}$ In Eqs. (2.1)-(2.5) the notations of Ref. 8(c) have been used: The dot represents a time derivative, $\nabla_{\alpha} \equiv \partial / \partial x_{\alpha}, G_{e_{n} e_{n}}\left(\vec{x}, \vec{x}^{\prime}\right)$ denotes the equilibrium correlation $\left\langle\widehat{e}_{n}(\vec{x}) \hat{e}_{n}\left(\vec{x}^{\prime}\right)\right\rangle_{0}$, and $K_{e_{n} e_{n}}\left(\vec{x}, \vec{x}^{\prime}\right)$ its inverse, $K_{n n}\left(\vec{x}, \vec{x}^{\prime}\right)$ being similarly the inverse of the densitydensity equilibrium correlation function while $\psi(\omega)=i Q(\omega-Q L Q)^{-1} Q$ is the resolvent operator for the motion orthogonal to the conserved densities which are projected out by means of the projection operator $Q=I-P$, while $L$ is the Liouville operator and a summation over repeated greek indices is implied. The basic approximation introduced below concerns the spatial nonlo- cality of the evolution equations (2.2) and (2.3). It will be assumed that all kernels in Eqs. (2.2) and (2.3) describe sufficiently short-ranged spatial correlations so that after a straightforward truncated Taylor expansion these evolution equations can be localized in space with, at most, second-order spatial gradients appearing.

\section{ELASTICITY OF NONUNIFORM FLUIDS}

Liquids $^{10}$ as well as liquid surfaces ${ }^{8(b)}$ are known to exhibit elastic properties. Here we show how the basic equations of elasticity result from the general evolution equations (2.1)-(2.3) in the limit where the evolution operator $\psi(\omega)$ can be approximated by its exact high-frequency limit

$$
\lim _{|\omega| \rightarrow \infty}-i \omega \psi(\omega)=Q
$$

or equivalently $\psi(\omega) \simeq i Q / \omega$, where $Q$ is the projection operator on the space orthogonal to the conserved densities. Eliminating the density $\delta n(\overrightarrow{\mathrm{x}}, \omega)$ from Eq. (2.2) with the aid of the continuity equation (2.1) and multiplying Eq. (2.2) by $i \omega$, we obtain with the use of Eq. (3.1)

$$
\begin{array}{r}
\omega^{2} m \rho(\overrightarrow{\mathrm{x}}) u_{\alpha}(\overrightarrow{\mathrm{x}}, \omega)+k_{B} T \rho(\overrightarrow{\mathrm{x}}) \nabla_{\alpha} \int d \overrightarrow{\mathrm{x}}^{\prime} K_{n n}\left(\overrightarrow{\mathrm{x}}, \overrightarrow{\mathrm{x}}^{\prime}\right) \nabla_{\beta}^{\prime}\left[\rho\left(\overrightarrow{\mathrm{x}}^{\prime}\right) u_{\beta}\left(\overrightarrow{\mathrm{x}}^{\prime}, \omega\right)\right]-\frac{1}{k_{B} T} \int d \overrightarrow{\mathrm{x}}^{\prime}\left\langle\hat{\mathrm{g}}_{\alpha}(\overrightarrow{\mathrm{x}}) Q \hat{\mathrm{g}}_{\beta}\left(\overrightarrow{\mathrm{x}}^{\prime}\right)\right\rangle u_{\beta}\left(\overrightarrow{\mathrm{x}}^{\prime}, \omega\right) \\
-\frac{i \omega}{k_{B} T^{2}} \int d \overrightarrow{\mathrm{x}}^{\prime}\left\langle\hat{\mathrm{g}}_{\alpha}(\overrightarrow{\mathrm{x}}) \delta \hat{e}_{n}\left(\overrightarrow{\mathrm{x}}^{\prime}\right)\right\rangle_{0} \delta T\left(\overrightarrow{\mathrm{x}}^{\prime}, \omega\right)=O(1 / \omega) .
\end{array}
$$

In Eq. (3.2) we have already taken into account that $\left\langle\dot{\hat{g}}_{\alpha}(\vec{x}) Q \delta \hat{e}_{n}\left(\vec{x}^{\prime}\right)\right\rangle_{0}$ and $\left\langle Q \hat{g}_{\alpha}(\vec{x})\right\rangle_{0}$ vanish identically because of the time-reversal symmetry of the equilibrium ensemble [as can be easily checked by explicit evaluation and using the value of $Q=I-P$ given in Ref. 8(c)]. Proceeding similarly for the energy equation (2.3) we obtain 


$$
\omega^{2} \rho(\vec{x}) \delta \epsilon(\vec{x}, \omega)-\frac{1}{k_{B} T^{2}} \int d \vec{x}^{\prime}\left\langle\delta \hat{\hat{e}}_{n}(\vec{x}) Q \delta \hat{\hat{e}}_{n}\left(\vec{x}^{\prime}\right)\right\rangle_{0} \delta T\left(\vec{x}^{\prime}, \omega\right)+\frac{i \omega}{k_{B} T} \int d \vec{x}^{\prime}\left\langle\delta \hat{e}_{n}(\vec{x}) \hat{g}_{\alpha}\left(\vec{x}^{\prime}\right)\right\rangle_{0} u_{\alpha}\left(\vec{x}^{\prime}, \omega\right)=O(1 / \omega)
$$

where we have taken into account that $\left\langle Q \delta \hat{e}_{n}(\vec{x})\right\rangle_{0}=0$ and $\left\langle\delta \hat{e}_{n}(\vec{x}) Q \hat{g}_{\alpha}\left(\vec{x}^{\prime}\right)\right\rangle_{0}=0$. Notice, moreover, that to order $1 / \omega$ the transient terms $\left\langle\psi(\omega) \delta \hat{e}_{n}(\vec{x})\right\rangle_{0}$ and $\left\langle\psi(\omega) \hat{\hat{g}}_{\alpha}(\vec{x})\right\rangle_{0}$ disappear from the evolution equations which now become local in time. An important further simplification occurs if in the third term of Eq. (3.2) we substitute $Q$ by its value $I-P$ and notice that $P$ is the sum of five projection operators $P=P_{n}+P_{e_{n}}+\Sigma_{\alpha} P_{g_{\alpha}}$, one for each conserved density. Because of the time-reversal symmetry of the equilibrium ensemble the contributions of $\boldsymbol{P}_{\boldsymbol{g}_{\alpha}}$ vanish identically. The contribution of $\boldsymbol{P}_{n}$ instead compensates exactly the second term of Eq. (3.2). Equation (3.2) thus becomes

$$
\begin{aligned}
& \omega^{2} m \rho(\overrightarrow{\mathrm{x}}) u_{\alpha}(\overrightarrow{\mathrm{x}}, \omega)-\frac{1}{k_{B} T} \int d \overrightarrow{\mathrm{x}}^{\prime}\left\langle\hat{\mathrm{g}}_{\alpha}(\overrightarrow{\mathrm{x}}) \hat{\mathrm{g}}_{\beta}\left(\overrightarrow{\mathrm{x}}^{\prime}\right)\right\rangle_{0} u_{\beta}\left(\overrightarrow{\mathrm{x}}^{\prime}, \omega\right) \\
& \quad+\frac{1}{k_{B} T} \int d \overrightarrow{\mathrm{x}}^{\prime}\left\langle\hat{\mathrm{g}}_{\alpha}(\overrightarrow{\mathrm{x}}) P_{e_{n}} \hat{\mathrm{g}}_{\beta}\left(\overrightarrow{\mathrm{x}}^{\prime}\right)\right\rangle_{0} u_{\beta}\left(\overrightarrow{\mathrm{x}}^{\prime}, \omega\right) \\
& \quad-\frac{i \omega}{k_{B} T^{2}} \int d \overrightarrow{\mathrm{x}}^{\prime}\left\langle\hat{\mathrm{g}}_{\alpha}(\overrightarrow{\mathrm{x}}) \delta \hat{e}_{n}\left(\overrightarrow{\mathrm{x}}^{\prime}\right)\right\rangle_{0} \delta T\left(\overrightarrow{\mathrm{x}}^{\prime}, \omega\right)=O(1 / \omega),
\end{aligned}
$$

while using the expression of $P_{e_{n}}$ and Eq. (3.3) the last two terms in the left-hand side (lhs) of Eq. (3.4) can be combined resulting in an $O(1 / \omega)$ term $\left[\sim \delta T\left(\overrightarrow{\mathrm{x}}^{\prime}, \omega\right) / \omega\right]$, i.e.,

$$
\begin{array}{r}
\omega^{2} m \rho(\overrightarrow{\mathrm{x}}) u_{\alpha}(\overrightarrow{\mathrm{x}}, \omega)-\frac{1}{k_{B} T} \int d \overrightarrow{\mathrm{x}}^{\prime}\left\langle\hat{\mathrm{g}}_{\alpha}(\overrightarrow{\mathrm{x}}) \hat{\mathrm{g}}_{\beta}\left(\overrightarrow{\mathrm{x}}^{\prime}\right)\right\rangle_{0} u_{\beta}\left(\overrightarrow{\mathrm{x}}^{\prime}, \omega\right) \\
=O(1 / \omega)
\end{array}
$$

showing that to dominant order the temperature uncou- ples from the velocity field leaving us with the basic equations of elasticity

$$
\begin{aligned}
& \omega^{2} m \rho(\overrightarrow{\mathrm{x}}) u_{\alpha}(\overrightarrow{\mathrm{x}}, \omega) \\
& +\frac{1}{k_{B} T} \nabla_{\alpha^{\prime}} \int d \overrightarrow{\mathrm{x}}^{\prime}\left\langle\delta \hat{\sigma}_{\alpha \alpha^{\prime}}(\overrightarrow{\mathrm{x}}) \delta \widehat{\sigma}_{\beta \beta^{\prime}}\left(\overrightarrow{\mathrm{x}}^{\prime}\right)\right\rangle_{0} \nabla_{\beta^{\prime}}^{\prime} u_{\beta^{\prime}}\left(\overrightarrow{\mathrm{x}}^{\prime}, \omega\right) \\
& =0,
\end{aligned}
$$

where we have introduced the deviation $\delta \hat{\sigma}_{\alpha \beta}$ $=\hat{\sigma}_{\alpha \beta}-\left\langle\hat{\sigma}_{\alpha \beta}\right\rangle_{0}$ of the microscopic stress tensor $\hat{\sigma}_{\alpha \beta}(\vec{x})$ from its equilibrium value $\left\langle\hat{\sigma}_{\alpha \beta}(\vec{x})\right\rangle_{0}\left[\hat{g}_{\alpha}(\vec{x})+\nabla_{\beta} \delta \hat{\sigma}_{\alpha \beta}(\vec{x})\right.$ $=0]$, and integrated by parts over $\vec{x}^{\prime}$. We now spatially localize Eq. (3.6) by assuming short-ranged spatial correlations resulting in the final equations

$\omega^{2} m \rho(\overrightarrow{\mathrm{x}}) u_{\alpha}(\overrightarrow{\mathrm{x}}, \omega)+\nabla_{\alpha^{\prime}}\left[c_{\alpha \alpha^{\prime} \beta \beta^{\prime}}(\overrightarrow{\mathrm{x}}) \nabla_{\beta^{\prime}} u_{\beta}(\overrightarrow{\mathrm{x}}, \omega)\right]=0$

where $c_{\alpha \alpha^{\prime} \beta \beta^{\prime}}(\vec{x})$ are the local elastic moduli ${ }^{8(b)}$ of the nonuniform fluid

$$
c_{\alpha \alpha^{\prime} \beta \beta^{\prime}}(\overrightarrow{\mathrm{x}})=\frac{1}{k_{B} T} \int d \overrightarrow{\mathrm{x}}^{\prime}\left\langle\delta \hat{\sigma}_{\alpha \alpha^{\prime}}(\overrightarrow{\mathrm{x}}) \delta \widehat{\sigma}_{\beta \beta^{\prime}}\left(\overrightarrow{\mathrm{x}}^{\prime}\right)\right\rangle_{0} .
$$

Note that these are the adiabatic elastic moduli. In order to obtain the isothermal moduli we repeat the above treatment with the additional approximation that the perturbations are isothermal, i.e., $\delta T(\vec{x}, \omega)=0$ in the general evolution equation (2.2) or equivalently in Eq. (3.4). This leads again to an equation of the type (3.7) but now featuring the isothermal moduli $c_{\alpha \alpha^{\prime} \beta \beta^{\prime}}^{(T)}$ instead of the adiabatic moduli $c_{\alpha \alpha^{\prime} \beta \beta}$ where the local isothermal elastic moduli $c_{\alpha \alpha^{\prime} \beta \beta^{\prime}}^{(T)}$ are defined as

$$
c_{\alpha \alpha^{\prime} \beta \beta^{\prime}}^{(T)}(\overrightarrow{\mathrm{x}})=c_{\alpha \alpha^{\prime} \beta \beta^{\prime}}(\overrightarrow{\mathrm{x}})-\frac{1}{k_{B} T} \int d \overrightarrow{\mathrm{x}}^{\prime} \int d \overrightarrow{\mathrm{x}}_{1} \int d \overrightarrow{\mathrm{x}}_{2}\left\langle\delta \hat{\sigma}_{\alpha \alpha^{\prime}}(\overrightarrow{\mathrm{x}}) \delta \widehat{e}_{n}\left(\overrightarrow{\mathrm{x}}_{1}\right)\right\rangle_{0} K_{e_{n} e_{n}}\left(\overrightarrow{\mathrm{x}}_{1}, \overrightarrow{\mathrm{x}}_{2}\right)\left\langle\delta \hat{e}_{n}\left(\overrightarrow{\mathrm{x}}_{2}\right) \delta \hat{\sigma}_{\beta \beta^{\prime}}\left(\overrightarrow{\mathrm{x}}^{\prime}\right)\right\rangle_{0},
$$

the distinction between both types of moduli being important only when both $\alpha=\alpha^{\prime}$ and $\beta=\beta^{\prime}$, since otherwise the second term in the right-hand side (rhs) of Eq. (3.9) will vanish identically.

\section{PLANAR LIQUID-VAPOR INTERFACE}

We now specialize Eq. (3.7) to the case where the nonuniform equilibrium fluid consists of a planar liquidvapor interface localized around the $x_{1}=0$ plane and separating a bulk liquid phase $\left(x_{1}<0\right)$ from a bulk vapor phase $\left(x_{1}>0\right)$. Because of the cylindrical rotational symmetry of this system only five elastic moduli are nonvanishing and linearly independent. ${ }^{8(b)}$ Using the Voigt notation $^{8(\mathrm{~b})} \quad c_{i j}=c_{\alpha \alpha^{\prime} \beta \beta^{\prime}}$ with $i=\left(\alpha, \alpha^{\prime}\right)$ and $j=\left(\beta, \beta^{\prime}\right)$ denoting a pair of indices [with $i=1=(1,1), i=2=(2,2)$, $i=3=(3,3), i=4=(1,2), i=5=(1,3)$, and $i=6=(2,3)]$, the nonvanishing moduli $c_{i j}=c_{i j}(\vec{x})$ are $c_{11}$, $c_{12}=c_{13}=c_{21}=c_{31}, c_{22}=c_{33}, c_{23}=c_{32}, c_{44}=c_{55}$, and $c_{66}$ together with the Cauchy relation $c_{23}=c_{22}-2 c_{66}$ [see Ref. 8(b) for a detailed discussion of these symmetry properties]. Hence for a planar liquid-vapor interface, Eq. (3.7) becomes

$$
\omega^{2} m \rho\left(x_{1}\right) u_{1}+\nabla_{1}\left[c_{11}\left(x_{1}\right) \nabla_{1} u_{1}\right]+\nabla_{1}\left[c_{12}\left(x_{1}\right)\left(\nabla_{2} u_{2}+\nabla_{3} u_{3}\right)\right]+c_{44}\left(x_{1}\right)\left[\left(\nabla_{2}^{2}+\nabla_{3}^{2}\right) u_{1}+\nabla_{1}\left(\nabla_{2} u_{2}+\nabla_{3} u_{3}\right)\right]=0
$$




$$
\omega^{2} m \rho\left(x_{1}\right) u_{2}+\nabla_{1}\left[c_{44}\left(x_{1}\right)\left(\nabla_{1} u_{2}+\nabla_{2} u_{1}\right)\right]+c_{12}\left(x_{1}\right) \nabla_{2} \nabla_{1} u_{1}+c_{22}\left(x_{1}\right) \nabla_{2}^{2} u_{2}+c_{23}\left(x_{1}\right) \nabla_{2} \nabla_{3} u_{3}+c_{66}\left(x_{1}\right) \nabla_{3}\left(\nabla_{2} u_{3}+\nabla_{3} u_{2}\right)=0 \text {, }
$$

$$
\omega^{2} m \rho\left(x_{1}\right) u_{3}+\nabla_{1}\left[c_{55}\left(x_{1}\right)\left(\nabla_{1} u_{3}+\nabla_{3} u_{1}\right)\right]+c_{12}\left(x_{1}\right) \nabla_{3} \nabla_{1} u_{1}+c_{23}\left(x_{1}\right) \nabla_{3} \nabla_{2} u_{2}+c_{22}\left(x_{1}\right) \nabla_{3}^{2} u_{3}+c_{66}\left(x_{1}\right) \nabla_{2}\left(\nabla_{2} u_{3}+\nabla_{3} u_{2}\right)=0 \text {, }
$$

where $u_{\alpha}$ denotes $u_{\alpha}(\vec{x}, \omega)$ and because of the translational symmetry parallel to the interface, all $c_{i j}(\overrightarrow{\mathrm{x}})$ and $\rho(\overrightarrow{\mathrm{x}})$ depend only on $x_{1}$. When $x_{1}$ belongs to a uniform bulk phase ( $\left|x_{1}\right|>\epsilon$, for instance) the local moduli $c_{i j}\left(x_{1}\right)$ become constants independent of $x_{1}$ and because of the spherical rotational symmetry of the bulk phases we have moreover $c_{11}=c_{22}=c_{33}, c_{44}=c_{55}=c_{66}$, and $c_{12}=c_{23}$, i.e., there are only two linearly independent nonvanishing moduli for either bulk phase. When $x_{1}$ belongs to the interfacial region we integrate Eqs. (4.1)-(4.3) through the interface from one bulk phase to the other. For the planar interface, localized between the $x_{1}=\epsilon$ and $x_{1}=-\epsilon$ planes, we meet three typical situations which can be summarized as follows:

$$
\int_{-\epsilon}^{\epsilon} d x_{1} \nabla_{1}\left[f\left(x_{1}\right) g(\overrightarrow{\mathrm{x}})\right]=\left[f\left(x_{1}\right) g(\overrightarrow{\mathrm{x}})\right]_{x_{1}=-\epsilon}^{x_{1}=\epsilon},
$$

$$
\begin{aligned}
& \int_{-\epsilon}^{\epsilon} d x_{1} f\left(x_{1}\right) \nabla_{1} g(\overrightarrow{\mathrm{x}})=0, \\
& \int_{-\epsilon}^{\epsilon} d x_{1} f\left(x_{1}\right) \nabla_{\alpha} g(\overrightarrow{\mathrm{x}})=\bar{f}\left[\nabla_{\alpha} g(\overrightarrow{\mathrm{x}})\right]_{x_{1}=0}, \quad \alpha \neq 1
\end{aligned}
$$

where $g(\vec{x})$ denotes a slowly varying function of $\vec{x}$ such as $u_{\alpha}(\vec{x}, \omega)$ or $\nabla_{\beta} u_{\alpha}(\vec{x}, \omega)(\beta \neq 1)$, while $f\left(x_{1}\right)$ denotes the remaining factor containing $c_{i j}\left(x_{1}\right)$ or $\rho\left(x_{1}\right)$. Equation (4.4) is, mathematically speaking, not an approximation since it simply tells us that by integrating a normal derivative through the interface we obtain the jump experienced by the corresponding quantity between the two bulk phases. As will be seen below, when all remaining terms are small, terms of this type transform the evolution equations into boundary conditions for the bulk quantities. Integrating by parts the lhs of Eq. (4.5) and using the slow variation of $g(\vec{x})$ in the domain $\left|x_{1}\right|<\epsilon$, we obtain

$$
\begin{aligned}
\int_{-\epsilon}^{\epsilon} d x_{1} f\left(x_{1}\right) \nabla_{1} g(\overrightarrow{\mathrm{x}}) & =\left[f\left(x_{1}\right) g(\overrightarrow{\mathrm{x}})\right]_{x_{1}=-\epsilon}^{x_{1}=\epsilon}-\int_{-\epsilon}^{\epsilon} d x_{1} g(\overrightarrow{\mathrm{x}}) \nabla_{1} f\left(x_{1}\right) \\
& \left.\simeq g(\overrightarrow{\mathrm{x}})\right|_{x_{1}=0}\left[f\left(x_{1}\right)\right]_{x_{1}=-\epsilon}^{x_{1}=\epsilon}-\left.g(\overrightarrow{\mathrm{x}})\right|_{x_{1}=0} \int_{-\epsilon}^{\epsilon} d x_{1} \nabla_{1} f\left(x_{1}\right),
\end{aligned}
$$

and hence Eq. (4.5). Finally, in Eq. (4.6) we have used the assumed slow variation of $\nabla_{a} g(\vec{x})$ to approximate it by its value inside the interfacial region $\left(\epsilon>\left|x_{1}\right| \simeq 0\right)$ and eliminated the $\epsilon$ dependence by approximating $\int_{-\epsilon}^{\epsilon} d x_{1} f\left(x_{1}\right)$ by its Gibb's surface excess value $\bar{f}$ :

$$
\begin{aligned}
\int_{-\epsilon}^{\epsilon} d x_{1} f\left(x_{1}\right) & =\int_{-\epsilon}^{\epsilon} d x_{1}\left[f\left(x_{1}\right)-\Theta_{+}\left(x_{1}\right) f\left(x_{1}=\infty\right)-\Theta_{-}\left(x_{1}\right) f\left(x_{1}=-\infty\right)\right]+\epsilon\left[f\left(x_{1}=\infty\right)+f\left(x_{1}=-\infty\right)\right] \\
& \simeq \bar{f}+O(\epsilon)
\end{aligned}
$$

with

$$
\bar{f}=\int_{-\infty}^{\infty} d x_{1}\left[f\left(x_{1}\right)-\Theta_{+}\left(x_{1}\right) f\left(x_{1}=\infty\right)-\Theta_{-}\left(x_{1}\right) f\left(x_{1}=-\infty\right)\right],
$$

where $\Theta_{ \pm}\left(x_{1}\right)$ are step functions $\left(=1\right.$ when $\pm x_{1}>0$, and zero otherwise), while $\epsilon$ has been considered as a small quantity. More details about this procedure can be found in Ref. 8(c) but the basic principles to be applied here are contained in the above results [Eqs. (4.4)-(4.6)]. Integrating Eqs. (4.1)-(4.3) through the interface and using Eqs. (4.4)-(4.6) we now obtain the following "surface equations":

$$
\begin{aligned}
& \omega^{2} m \bar{\rho} u_{1}+\bar{c}_{44}\left(\nabla_{2}^{2}+\nabla_{3}^{2}\right) u_{1} \\
& =\left[c_{11} \nabla_{1} u_{1}+c_{12}\left(\nabla_{2} u_{2}+\nabla_{3} u_{3}\right)\right]_{+}^{-}, \\
& \omega^{2} m \bar{\rho} u_{2}+\bar{c}_{22} \nabla_{2}^{2} u_{2}+\bar{c}_{23} \nabla_{2} \nabla_{3} u_{3}+\bar{c}_{66} \nabla_{3}\left(\nabla_{3} u_{2}+\nabla_{2} u_{3}\right) \\
& =\left[c_{44}\left(\nabla_{1} u_{2}+\nabla_{2} u_{1}\right)\right]_{+}^{-}, \\
& \omega^{2} m \bar{\rho} u_{3}+\bar{c}_{22} \nabla_{3}^{2} u_{3}+\bar{c}_{23} \nabla_{3} \nabla_{2} u_{2}+\bar{c}_{66} \nabla_{2}\left(\nabla_{3} u_{2}+\nabla_{2} u_{3}\right) \\
& =\left[c_{55}\left(\nabla_{1} u_{3}+\nabla_{3} u_{1}\right)\right]_{+}^{-} \text {, }
\end{aligned}
$$

where $u_{\alpha} \equiv u_{\alpha}\left(x_{1}=0, x_{2}, x_{3} ; \omega\right)$, while for instance $[a]_{+}^{-}=a\left(x_{1}=-0, x_{2}, x_{3}\right)-a\left(x_{1}=+0, x_{2}, x_{3}\right)$ denotes the jump of the magnitude $a(\vec{x})$ when going from one bulk phase to the other across the interfacial region. The surface equations (4.10)-(4.12) are much simpler than the original equations $(4.1)-(4.3)$ since the rapidly variable coefficients $\rho\left(x_{1}\right)$ and $c_{i j}\left(x_{1}\right)$ have been eliminated in favor of the surface excess values $\bar{\rho}$ and $\bar{c}_{i j}$ defined by Eq. (4.9), which are constants. The lhs of Eqs. (4.10)-(4.12) resemble the equations describing the elasticity of a thin film for which the bulk properties in the rhs appear as source terms connecting the bulk and surface phases. Equations (4.10)-(4.12) will be used in Sec. V to study the effect of the surface structure (buried in the surface excess values) on the elastic surface waves. Putting all surface excess values equal to zero $\left(\bar{\rho}=0, \bar{c}_{i j}=0\right)$ and neglecting the bulk vapor phase one easily recovers from Eqs. 
(4.10)-(4.12) the free surface boundary conditions of the macroscopic theory of elasticity. ${ }^{1}$ This is most easily done by switching from the velocity field $u_{\alpha}(\overrightarrow{\mathrm{x}}, \omega)$ to the displacement field $d_{\alpha}(\overrightarrow{\mathrm{x}}, \omega)$ with $u_{\alpha}(\overrightarrow{\mathrm{x}}, \omega)=-i \omega d_{\alpha}(\overrightarrow{\mathrm{x}}, \omega)$ and writing Hooke's law in the Voigt notation as $\sigma_{i}=\Sigma_{j} c_{i j} s_{i}$ with $\sigma_{i}$ denoting the $(\alpha, \beta)=i$ component of the macroscopic stress tensor and $s_{i}=\left(\nabla_{\alpha} d_{\beta}+\nabla_{\beta} d_{\alpha}\right) / 2$ the $i=(\alpha, \beta)$ component of the macroscopic strain tensor. At a free surface the normal stresses have to vanish, i.e., $\sigma_{i}=0$ for $i=(1, \alpha)$, which when expressed in terms of the elastic moduli $c_{i j}$ corresponds to the vanishing of the rhs of Eqs. (4.10)-(4.12). The interfacial structure, contained in the lhs of Eqs. (4.10)-(4.12) can hence be seen to act as source terms for the macroscopic theory of elasticity.

\section{ELASTIC SURFACE WAVES}

As discussed in Sec. IV, the elastic perturbations of the displacement $\left[d_{\alpha}(\overrightarrow{\mathrm{x}}, \omega)\right]$ or velocity field $\left[u_{\alpha}(\overrightarrow{\mathrm{x}}, \omega)\right.$ $\left.=-i \omega d_{\alpha}(\overrightarrow{\mathrm{x}}, \omega)\right]$ of a liquid-vapor system with a planar equilibrium interface are described by Eqs. (4.1)-(4.3) which in the interfacial region can be approximated by Eqs. (4.10)-(4.12). In order to extract further useful information from these equations we now consider planewave perturbations of the liquid neglecting the vapor phase, i.e., we consider a free liquid surface.

\section{A. Bulk perturbations}

We first look for plane-wave solutions $u_{\alpha}(\overrightarrow{\mathrm{x}}, \omega) \sim \exp [i(\overrightarrow{\mathrm{k}} \cdot \overrightarrow{\mathrm{x}}-\omega t)]$ of Eqs. (4.1)-(4.3) when $x_{1}$ belongs to the bulk liquid phase where all elastic moduli become constants $c_{i j}\left(x_{1}\right)=c_{i j}^{(-)}\left(x_{1}\right) \equiv c_{i j}$. Since the bulk liquid phase is spherically symmetric we can without loss of generality put $\overrightarrow{\mathrm{k}}$ in the $x_{2}$ direction, i.e., $u_{\alpha}(\vec{x}, \omega)=u_{\alpha} \exp \left[i\left(k x_{2}-\omega t\right)\right]$. Equations (4.1)-(4.3) now yield (for $x_{1}<0$ )

$$
\begin{aligned}
& \omega^{2} m \rho u_{1}-c_{44} k^{2} u_{1}=0, \\
& \omega^{2} m \rho u_{2}-c_{22} k^{2} u_{2}=0, \\
& \omega^{2} m \rho u_{3}-c_{66} k^{2} u_{3}=0,
\end{aligned}
$$

where $\rho$ denotes the density of the liquid bulk phase and $c_{22}, c_{44}, c_{66}$ its (for instance, adiabatic) elastic moduli. Because of the isotropy of the bulk phase we must have moreover $c_{44}=c_{66}$. Equations (5.1)-(5.3) thus predict a longitudinal elastic phonon $\left(\omega^{2}=c_{l}^{2} k^{2} ; c_{l}^{2}=c_{22} / m \rho ; u_{2}|| k\right)$ and two identical transverse elastic phonons $\left(\omega^{2}=c_{t}^{2} k^{2} ; c_{t}^{2}=c_{44} / m \rho=c_{66} / m \rho ; u_{1}, u_{3} \perp k\right)$ in agreement with the macroscopic theory. ${ }^{1}$ The general solution of the bulk equations can thus be written as a superposition of these elastic phonons, i.e., for an arbitrary geometry we will have

$$
\begin{aligned}
& \overrightarrow{\mathrm{u}}(\overrightarrow{\mathrm{x}}, \omega)=\overrightarrow{\mathrm{u}} e^{i\left(\overrightarrow{\mathrm{k}}_{l} \cdot \overrightarrow{\mathrm{x}}-\omega t\right)}+\overrightarrow{\mathrm{u}}^{\prime} e^{i\left(\overrightarrow{\mathrm{k}}_{t} \cdot \overrightarrow{\mathrm{x}}-\omega t\right)}, x_{1}<0 \\
& \overrightarrow{\mathrm{k}}_{l} \times \overrightarrow{\mathrm{u}}=0 ; \quad \overrightarrow{\mathrm{k}}_{t} \cdot \overrightarrow{\mathrm{u}}^{\prime}=0, \\
& k_{l}^{2}=\omega^{2} / c_{l}^{2} ; \quad k_{t}^{2}=\omega^{2} / c_{t}^{2} .
\end{aligned}
$$

A similar result also holds for the vapor phase $\left(x_{1}>0\right)$, but since we will neglect the vapor phase we can simply put $\overrightarrow{\mathrm{u}}(\overrightarrow{\mathrm{x}}, \omega)$ equal to zero for $x_{1}>0$.

\section{B. Surface equations}

In the interfacial region $x_{1} \simeq 0$ the bulk perturbation (5.4) has to satisfy, moreover, the surface equations (4.10)- (4.12). Let us introduce the notation $\overrightarrow{\mathrm{x}}=\left(x_{1}, \overrightarrow{\mathrm{r}}\right)$, $\overrightarrow{\mathrm{k}}=\left(k_{1}, \overrightarrow{\mathrm{q}}\right)$ with $\overrightarrow{\mathrm{r}}=\left(x_{2}, x_{3}\right)$ and $\overrightarrow{\mathrm{q}}=\left(k_{2}, k_{3}\right)$ being the components of $\vec{x}$ and $\vec{k}$ in the interface plane $\left(x_{1}=0\right)$. Since we are interested only in surface waves we look for solutions which are spatially damped inside the bulk liquid phase, i.e., plane waves with $k_{1}=-i \kappa$, where the real part of $\kappa$ is strictly positive: $\exp (i \overrightarrow{\mathrm{k}} \cdot \overrightarrow{\mathbf{x}})$ $=\exp \left(i \overrightarrow{\mathrm{q}} \cdot \overrightarrow{\mathrm{r}}+\kappa x_{1}\right), x_{1}<0$. The general perturbation (5.4) for surface plane waves reads thus in this notation:

$u_{\alpha}(\overrightarrow{\mathrm{x}}, \omega)=\left(u_{\alpha} e^{\kappa_{l} x_{1}}+u_{\alpha}^{\prime} e^{\kappa_{t} x_{1}}\right) e^{i(\overrightarrow{\mathrm{q}} \cdot \overrightarrow{\mathrm{r}}-\omega t)}, \quad x_{1}<0$

where the values of $\kappa_{l}$ and $\kappa_{t}$ are fixed by the bulk dispersion relations $\left[\omega^{2}=c_{\alpha}^{2} k^{2}=c_{\alpha}^{2}\left(q^{2}-\kappa_{\alpha}^{2}\right) ; \alpha=(l, t)\right]$ as

$$
\begin{aligned}
& \kappa_{l}=\left(q^{2}-\omega^{2} / c_{l}^{2}\right)_{+}^{1 / 2}, \\
& \kappa_{t}=\left(q^{2}-\omega^{2} / c_{t}^{2}\right)_{+}^{1 / 2},
\end{aligned}
$$

where the subscript indicates that the square roots have to be taken with positive real part. Because of the isotropy in the $\vec{r}$ plane we can without loss of generality put $\vec{q}$ in the $x_{2}$ direction $\left[\exp (i \overrightarrow{\mathrm{q}} \cdot \overrightarrow{\mathrm{r}})=\exp \left(i q x_{2}\right)\right]$. Substituting (5.5) in the surface equations we obtain (neglecting the vapor phase)

$$
\begin{aligned}
\left(\omega^{2} m \bar{\rho}-\bar{c}_{44} q^{2}\right)\left(u_{1}+u_{1}^{\prime}\right)= & c_{11}\left(\kappa_{l} u_{1}+\kappa_{t} u_{1}^{\prime}\right) \\
& +i q c_{12}\left(u_{2}+u_{2}^{\prime}\right), \\
\left(\omega^{2} m \bar{\rho}-\bar{c}_{22} q^{2}\right)\left(u_{2}+u_{2}^{\prime}\right)= & c_{44}\left(\kappa_{l} u_{2}+\kappa_{t} u_{2}^{\prime}\right) \\
& +i q c_{44}\left(u_{1}+u_{1}^{\prime}\right), \\
\left(\omega^{2} m \bar{\rho}-\bar{c}_{66} q^{2}\right)\left(u_{3}+u_{3}^{\prime}\right)= & c_{44}\left(\kappa_{l} u_{3}+\kappa_{t} u_{3}^{\prime}\right),
\end{aligned}
$$

whereas the tranversality condition $\vec{\kappa}_{t} \cdot \overrightarrow{\mathrm{u}}^{\prime}=0$ of (5.4) becomes

$$
q u_{2}^{\prime}=i \kappa_{t} u_{1}^{\prime}
$$

while $\vec{\kappa}_{l} \times \overrightarrow{\mathbf{u}}=0$ leads to

$$
\begin{aligned}
& q u_{1}=-i \kappa_{l} u_{2}, \\
& q u_{3}=0 ; \kappa_{l} u_{3}=0,
\end{aligned}
$$

where Eq. (5.13) implies that $u_{3}=0$ since for surface waves both $q$ and $\kappa_{l}$ have to be different from zero. The bulk perturbation (5.5) will hence satisfy the surface equations if a nontrivial solution to Eqs. (5.8)-(5.13) can be found.

\section{Dispersion relation for surface waves}

As seen from Eqs. (5.10) and (5.13), $u_{3}$ and $u_{3}^{\prime}$ uncouple from the remaining components leading immediately to a nontrivial solution $u_{3}=0, u_{3}^{\prime} \neq 0$ provided the following dispersion relation is satisfied: 


$$
\omega^{2} m \bar{\rho}-\bar{c}_{66} q^{2}=c_{44} \kappa_{t} .
$$

This solution which describes transverse motions in the plane of the interface $\left(u_{3}^{\prime} \perp q, u_{3}^{\prime} \neq 0\right)$ is the result of the existence of a surface structure and as such does not appear in the macroscopic theory. ${ }^{1}$ Indeed when the excess values $\bar{\rho}$ and $\bar{c}_{66}$ are neglected in Eq. (5.14), the latter is seen to imply $\kappa_{t}=0$ which is impossible for surface waves implying, in turn, that $u_{3}^{\prime}=0$. The new solution $\left(u_{3}^{\prime} \neq 0\right)$ is possible only when $\bar{\rho}$ or $\bar{c}_{66}$ are finite as the result of the existence of a surface structure.

The remaining four equations (5.8) and (5.9), and (5.11) and (5.12) couple $\left(u_{1}, u_{2}\right)$ to $\left(u_{1}^{\prime}, u_{2}^{\prime}\right)$ and hence describe mixed longitudinal-transverse motions occurring in the sagittal plane (i.e., the plane normal to the interface and containing the wave vector $\overrightarrow{\mathrm{q}}$ ). A nontrivial solution of these four equations can be found only when all four components $u_{1}, u_{2}, u_{1}^{\prime}, u_{2}^{\prime}$ are different from zero [because $u_{1}^{\prime} / u_{2}^{\prime}$ and $u_{1} / u_{2}$ are fixed by Eqs. (5.11) and (5.12)] and when, moreover, the following dispersion relation is satisfied:

$$
\begin{gathered}
\left(\omega^{2} m \bar{\rho}-\bar{c}_{44} q^{2}\right)\left(\omega^{2} m \bar{\rho}-\bar{c}_{22} q^{2}\right)\left(\kappa_{l} \kappa_{t}-q^{2}\right) \\
+m \rho \omega^{2}\left[\kappa_{l}\left(\omega^{2} m \bar{\rho}-\bar{c}_{44} q^{2}\right)+\kappa_{t}\left(\omega^{2} m \bar{\rho}-\bar{c}_{22} q^{2}\right)\right] \\
+c_{44}^{2}\left[\left(q^{2}+\kappa_{t}^{2}\right)^{2}-4 q^{2} \kappa_{l} \kappa_{t}\right]=0 .
\end{gathered}
$$

When all surface excess values $\left(\bar{\rho}, \bar{c}_{22}, \bar{c}_{44}\right)$ are neglected, Eq. (5.15) reduces to the last term in its lhs which corresponds exactly to the result of the macroscopic theory. ${ }^{1}$

\section{Approximate solutions}

Without a priori knowledge of the magnitude of the surface excess values, the general dispersion equation for transverse [Eq. (5.14)] and sagittal-plane [Eq. (5.15)] motions are difficult to solve. In view of this we will consider all surface excess terms as small and compute their influence on the results of the macroscopic theory ${ }^{1}$ by perturbation theory. It should be clear, however, that when the surface excess values are not small, different solutions may exist. Consider first the transverse surface waves determined by Eq. (5.14). Taking the square of this equation and substituting $\kappa_{t}$ by its value from Eq. (5.7) we obtain

$$
\omega^{2}=q^{2} c_{t}^{2}\left[1-\left[\frac{\omega^{2}}{q^{2} c_{t}^{2}} \frac{q \bar{\rho}}{\rho}-\frac{q \bar{c}_{66}}{c_{66}}\right]^{2}\right],
$$

and solving by iteration around the bulk transverse frequency $\left(\omega \simeq q c_{t}\right)$ we obtain

$$
\left.\omega^{2}=q^{2} c_{t}^{2}\left[1-(q \bar{\epsilon})^{2}\right], \quad \bar{\epsilon}=|| \frac{\bar{\rho}}{\rho}-\frac{\bar{c}_{66}}{c_{66}}\right) \mid,
$$

i.e., a propagating transverse surface wave at a frequency slightly below the transverse bulk frequency $\left(q c_{t}\right)$ and with a spatial damping coefficient $\kappa_{t}=q^{2} \bar{\epsilon}$. Observation of this surface wave could yield direct access to the surface structure via $\bar{\rho}$ or $\bar{c}_{66}$. We should not forget, however, that the precise location of the liquid surface $\left(x_{1}=0\right)$ should be done intrinsically and not with respect to the laboratory frame of reference. This is usually accomplished by defining the $x_{1}=0$ plane through Gibb's condition of zero absorption, $\bar{\rho}=0$, so that the surface acquires no inertia. It is equally possible to define the surface by the condition of zero surface elasticity, $\bar{c}_{66}=0$, in which case the surface will generally acquire some inertia $(\bar{\rho} \neq 0)$. In either case ( $\bar{\rho}=0$ or $\left.\bar{c}_{66}=0\right)$ Eq. (5.17) will yield experimental access to the surface structure through $\bar{c}_{66}$ or $\bar{\rho}$.

For the sagittal waves determined by Eq. (5.15) we similarly look for solutions in the vicinity of Rayleigh's surface waves ${ }^{1}$ occurring slightly below the bulk transverse frequency

$$
\omega^{2}=q^{2} c_{t}^{2}(\xi+\delta \xi)^{2},
$$

where $|\xi|<1$ and $\delta \xi$ is a small correction due to the surface structure. In the limit of vanishing surface excess values $\left(\bar{\rho}=0, \bar{c}_{22}=0, \bar{c}_{44}=0\right), \delta \xi=0$ and $\xi$ is determined from Eq. (5.15) as the solution of the equation

$$
\left(\xi^{2}-2\right)^{2}=4\left(1-\xi^{2}\right)^{1 / 2}\left(1-\frac{c_{t}^{2}}{c_{l}^{2}} \xi^{2}\right)^{1 / 2}
$$

which is identical to the result of the macroscopic theory. ${ }_{.}^{1}$ Treating all surface excess values as small we find $\delta \xi=q \bar{\lambda}$ with $\bar{\lambda}$ determined by

$$
\begin{aligned}
& \bar{\lambda}=\frac{\xi}{4}\left[\frac{\Delta\left(\frac{\bar{c}_{44}}{c_{44}}-\frac{\bar{\rho}}{\rho} \xi^{2}\right)+\Delta^{\prime}\left(\frac{\overline{c_{22}}}{c_{44}}-\frac{\bar{\rho}}{\rho} \xi^{2}\right)}{\left(\xi^{2}-2\right)+\frac{\Delta}{\Delta^{\prime}}+\frac{c_{t}^{2} \Delta^{\prime}}{c_{l}^{2} \Delta}}\right], \\
& \Delta=\left(1-\frac{c_{t}^{2}}{c_{l}^{2}} \xi^{2}\right)^{1 / 2}, \Delta^{\prime}=\left(1-\xi^{2}\right)^{1 / 2},
\end{aligned}
$$

where $\xi$ denotes the solution of Eq. (5.19) while the spatial damping coefficients are

$$
\kappa_{t}=q\left[1-(\xi+q \bar{\lambda})^{2}\right]^{1 / 2}
$$

and

$$
\kappa_{l}=q\left[1-c_{t}^{2}(\xi+q \bar{\lambda})^{2} / c_{l}^{2}\right]^{1 / 2} .
$$

Again the intrinsic result is obtained by localizing the interface with the aid of, for instance, Gibb's condition $\bar{\rho}=0$.

When the surface structure is retained, Eq. (5.15) can have solutions which do not appear in the macroscopic theory. This is seen, for example, by looking for solutions in the domain

$$
q^{2} c_{t}^{2} \ll<\omega^{2} \ll q^{2} c_{l}^{2}, \omega^{2} \ll q^{2} c_{t}^{2}\left(\bar{c}_{44} / \bar{c}_{22}\right)^{2}
$$

which, as will be shown in a later study, is the elastic equivalent of the domain where the capillary waves occur in the hydrodynamic theory. Treating again the surface excess values as small, we obtain this time

$$
\omega^{2}=\frac{q^{3} \bar{c}_{44}}{m \rho(1+q \bar{\rho} / \rho)},
$$

which is precisely the elastic equivalent of the capillary 
wave with $\bar{c}_{44}$ playing the role of the high-frequency surface tension. When $\bar{\rho}=0$ the spatial damping coefficients are $\kappa_{t}=q\left|1-q \bar{c}_{44} / c_{44}\right|$ and $\kappa_{l}=q\left|1-q \bar{c}_{44} / c_{22}\right|$. Note also that when $q \bar{\rho} / \rho \gg 1$, Eq. (5.22) predicts a phonon frequency $\left(\omega^{2}=q^{2} \bar{c}_{44} / m \bar{\rho}\right)$ which is formally equivalent to the condensation or crystallization waves found recently ${ }^{11}$ for interfaces which acquire an inertia as the result of some nonequilibrium conditions. In the equilibrium situation considered here it should be more realistic to consider Eq. (5.22) with $\bar{\rho}=0$. In any case we are not aware of any other theoretical prediction of capillary-wavelike $\left(\omega \sim q^{3 / 2}\right)$ elastic surface waves.

\section{CONCLUSIONS}

We have shown how the equations describing the elastic behavior of a nonuniform fluid can be obtained in a straightforward manner by taking the high-frequency limit of the exact Mori-Zwanzig evolution equations. Both adiabatic $[\delta T(\vec{x}, \omega) \neq 0]$ and isothermal $[\delta T(\vec{x}, \omega)=0]$ situations can be considered. In the adiabatic case the unique approximation to the microscopic evolution operator $\psi(\omega) \simeq i Q / \omega$ [based on the exact result $\left.\lim _{|\omega| \rightarrow \infty}-i \omega \psi(\omega)=Q\right]$ is sufficient to uncouple the temperature $[\delta T(\vec{x}, \omega)]$ from the velocity field $\left[u_{\alpha}(\vec{x}, \omega)\right]$. In both situations the velocity field [or equivalently the local displacement $\left.d_{\alpha}(\overrightarrow{\mathrm{x}}, \omega), u_{\alpha}(\overrightarrow{\mathrm{x}}, \omega)=-i \omega d_{\alpha}(\overrightarrow{\mathrm{x}}, \omega)\right]$ obeys, after spatial localization, a wave equation [Eq. (3.7)] involving local adiabatic or isothermal elastic moduli whose microscopic expression is given by Eqs. (3.8) and (3.9). When the nonuniform fluid consists of a liquid-vapor sys- tem with a planar equilibrium interface these equations can be reduced in the bulk phases to those of the macroscopic theory of elasticity ${ }^{1}$ and in the interfacial region to surface equations coupling the bulk and surface perturbations. When the latter perturbations are neglected the equilibrium conditions for a free surface as used in the macroscopic theory ${ }^{1}$ are recovered. The equations for the surface perturbations depend on the structure (profile) of the equilibrium surface through the appearance of surface excess elastic moduli. The structure of the surface can be made accessible to experimental investigation through the observation of the elastic surface waves. We find that the surface structure produces a small wave-vector-dependent shift in the frequency and spatial damping coefficient of Rayleigh's elastic surface wave [cf. Eqs. (5.18) - (5.20)]. We also find that in the presence of nonvanishing surface excess values, new surface waves, not predicted by the macroscopic theory, ${ }^{1}$ are possible. We have found a new transverse wave [Eq. (5.16)] occurring slightly below the frequency of the bulk transverse phonon and a new capillary-wavelike $\left(\omega \sim q^{3 / 2}\right)$ elastic surface wave [Eq. (5.22)]. These new surface waves are, to the best of our knowledge, predicted here for the first time. An important question left unanswered by the present investigation concerns the order of magnitude of the surface excess moduli involved. This point may be of importance for the experimental observability of these new surface waves.

\section{ACKNOWLEDGMENTS}

One of us (M.B.) is supported in part by the Fonds National de la Recherche Scientifique.
${ }^{1}$ L. D. Landau and E. M. Lifshitz, Theory of Elasticity (Pergamon, Oxford, 1975), Chap. 3.

2R. Evans, Adv. Phys. 28, 143 (1979); J. K. Percus, in Studies in Statistical Mechanics (North-Holland, Amsterdam, 1982), Vol. VIII.

3J. S. Rowlinson and B. Widom, Molecular Theory of Capillarity (Clarendon, Oxford, 1982).

${ }^{4}$ A. D. J. Haymet and D. W. Oxtoby, J. Chem. Phys. 74, 2559 (1981); M. Baus (unpublished).

${ }^{5}$ D. Ronis, D. Bedeaux, and I. Oppenheim, Physica (Utrecht) 90A, 487 (1978); D. Ronis and I. Oppenheim, ibid. 117A, 317 (1983); D. Ronis (unpublished).

${ }^{6}$ D. Bedeaux, A. M. Albano, and P. Mazur, Physica (Utrecht) $\underline{82 \mathrm{~A}}, 438$ (1976); D. Bedeaux and I. Oppenheim, ibid. 90A, 39 (1978).
${ }^{7}$ M. S. Jhon, R. C. Desai, and J. S. Dahler, Chem. Phys. Lett. 56, 151 (1978); J. Chem. Phys. 68, 5615 (1978); Adv. Chem. Phys. 46, 279 (1981).

${ }^{8}$ (a) M. Baus and C. F. Tejero, Chem. Phys. Lett. 84, 222 (1981); (b) M. Baus, J. Chem. Phys. 76, 2003 (1982); (c) M. Baus and C. F. Tejero, ibid. 78, 483 (1983).

${ }^{9}$ B. U. Felderhof, Physica (Utrecht) 48, 541 (1970); L. A. Turski and J. S. Langer, Phys. Rev. A 22, 2189 (1980); M. Grant and R. C. Desai, ibid. 25, 2727 (1982); J. Chem. Phys. 76, 5160 (1982); Phys. Rev. A 27, 2577 (1983).

${ }^{10}$ R. Zwanzig and R. D. Mountain, J. Chem. Phys. 43, 4464 (1965); P. Schofield, Proc. Phys. Soc. London 88, 149 (1966).

${ }^{11}$ L. Puech and B. Castaing, J. Phys. (Paris) Lett. 43, L601 (1982); A. I. Korotchenko and A. A. Samokhin, J. Phys. (Paris) Colloq. 41, C9-259 (1980). 